\title{
Article of Significant Interest in This Issue
}

A Missing Link Identified in the Regulation of Ser/Thr Phosphorylation in Escherichia coli

Long studied exclusively in eukaryotes, Ser/Thr phosphorylation is now recognized as a common modification in bacteria. However, both the mechanism of its regulation and its physiological significance remain largely mysterious, particularly in Gramnegative organisms. Unlike His or Asp phosphorylation, phosphorylation of Ser/Thr residues is a stable modification and a dedicated Ser/Thr phosphatase is required in order to enable reversible regulation. Rajagopalan and Dworkin (e00225-18) report the identification of the first protein phosphatase 2C-like Ser/Thr phosphatase in Escherichia coli. This study therefore provides a new foothold in efforts to understand the function of Ser/Thr phosphorylation in a model Gram-negative bacterium. 